\title{
Superior Mesenteric Artery Syndrome Accompanying With Nutcracker Syndrome: A Case Report
}

\author{
Mikail Inal ${ }^{1,} ;$ Birsen Unal Daphan ${ }^{1} ;$ Mirace Yasemin Karadeniz Bilgili ${ }^{1}$ \\ ${ }^{1}$ Department of Radiology, School of Medicine, Kirikkale University, Kirikkale, Turkey \\ *Corresponding Author: Mikail Inal, Department of Radiology, School of Medicine, Kirikkale University, Kirikkale, Turkey. Tel: +90-03182252489, Fax:+90-0318224786, E-mail:inal_m@ \\ hotmail.com
}

Received: September 10, 2013; Revised: March 10, 2014; Accepted: August 31, 2014

\begin{abstract}
Introduction:Superior mesentericartery syndrome is an uncommon cause of duodenal obstruction, and its manifestationsare generally associated with compression on the third part of the duodenum between the abdominal aorta and superior mesenteric artery.

Case Presentation:In this report, a patient is described presenting with epigastric pain and weight loss due to superior mesenteric artery syndrome. The patient has also nutcracker syndrome, which is the compression of the left renal vein between the aorta and the superior mesenteric artery at its origin.

Conclusions: In addition to an appropriate clinical history, CT findings indicating decreased aortomesenteric angle and a shortened aortomesenteric distance can suggest the diagnosis for both the superior mesenteric artery syndrome and accompanying nutcracker syndrome.
\end{abstract}

Keywords:Duodenal Obstruction; Weight Loss; Hematuria; Renal Nutcracker Syndrome; Superior Mesenteric Artery Syndrome

\section{Introduction}

Superior mesenteric artery (SMA) syndrome is a rare cause of abdominal pain, nausea, and vomiting that may be undiagnosed in patients presenting to the emergency department. SMA syndrome is arteriomesenteric obstruction of the duodenum, which is characterized by compression of the third portion of the duodenum by SMA (as it passes over this portion of the duodenum). Nutcracker syndrome (NCS) is characterized by impeded outflow from the left renal vein (LRV) into the inferior vena cava (IVC) due to extrinsic LRV compression. We presented this case report in order to discuss the diagnosis of a patient with both SMA syndrome and accompanying NCS with computed tomography (CT) findings.

\section{Case Presentation}

A 28-year-old man was admitted to Kirikkale University Medicine Faculty, Radiology Department, Kirikkale, Turkey, on $26^{\text {th }}$ April 2013. He had a history of 1-day vomiting, epigastric pain, and bloating (Table 1). He also reported weight loss, anorexia and intermittent abdominal pain during the past few years. Abdominal examination revealed upper abdominal tenderness and distension. Blood test results were as follows: mean corpuscular hemoglobin concentration, $36.3 \mathrm{~g} / \mathrm{dL}$; granulocyte (percentage, count), $\% 77,8 \times 10^{3} / \mathrm{uL}$; creatinine $0.61 \mathrm{mg} / \mathrm{dL}$, with no other abnormalities. Urinalysis revealed no hematuria.

\begin{tabular}{|c|c|}
\hline Patient Features & Age $=28 \mathrm{y}$, Gender $=$ Male \\
\hline Symptoms & $\begin{array}{l}\text { Vomiting, epigastric pain, bloating, } \\
\text { weight loss, anorexia, intermittent } \\
\text { abdominal pain }\end{array}$ \\
\hline $\begin{array}{l}\text { Duration of } \\
\text { symptoms, y }\end{array}$ & $>3-4$ \\
\hline Clinical findings & upper abdominal tenderness, distension \\
\hline Diagnostic testing & Blood tests, urinalysis, CT \\
\hline Blood tests & $\begin{array}{c}\text { MCHC, } 36.3 \mathrm{~g} / \mathrm{dL} \text {; granulocyte } \% 77 ; 8 \times 10^{3} / \\
\text { uL; creatinine } 0.61 \mathrm{mg} / \mathrm{dL}\end{array}$ \\
\hline Urinalysis & No hematuria \\
\hline CT findings & $\begin{array}{c}\text { Reduction of the aortomesenteric angle, } \\
\text { decreased aortomesenteric distance, } \\
\text { compression of LRV, gastric and proximal } \\
\text { duodenal dilatation }\end{array}$ \\
\hline $\begin{array}{l}\text { Comorbid } \\
\text { Condition }\end{array}$ & Colic abdominal pain, liver hemangiomas \\
\hline
\end{tabular}

The scan was performed with GE Hi-Speed, a dual-detector CT scanner, which is regularly calibrated. CT images showed the reduction of the angle between the abdominal aorta (AA) and SMA, and decreased aortomesenteric distance. The angle between the AA and SMA was $14.30^{\circ}$ (Figure 1A), and the aortomesenteric distance was $3.9 \mathrm{~mm}$

Copyright ( ) 2014, Iranian Red Crescent Medical Journal; Published by Kowsar. This is an open-access article distributed under the terms of the Creative Commons Attribution-NonCommercial 4.0 International License (http://creativecommons.org/licenses/by-nc/4.0/) which permits copy and redistribute the material just in noncommercial usages, provided the original work is properly cited. 

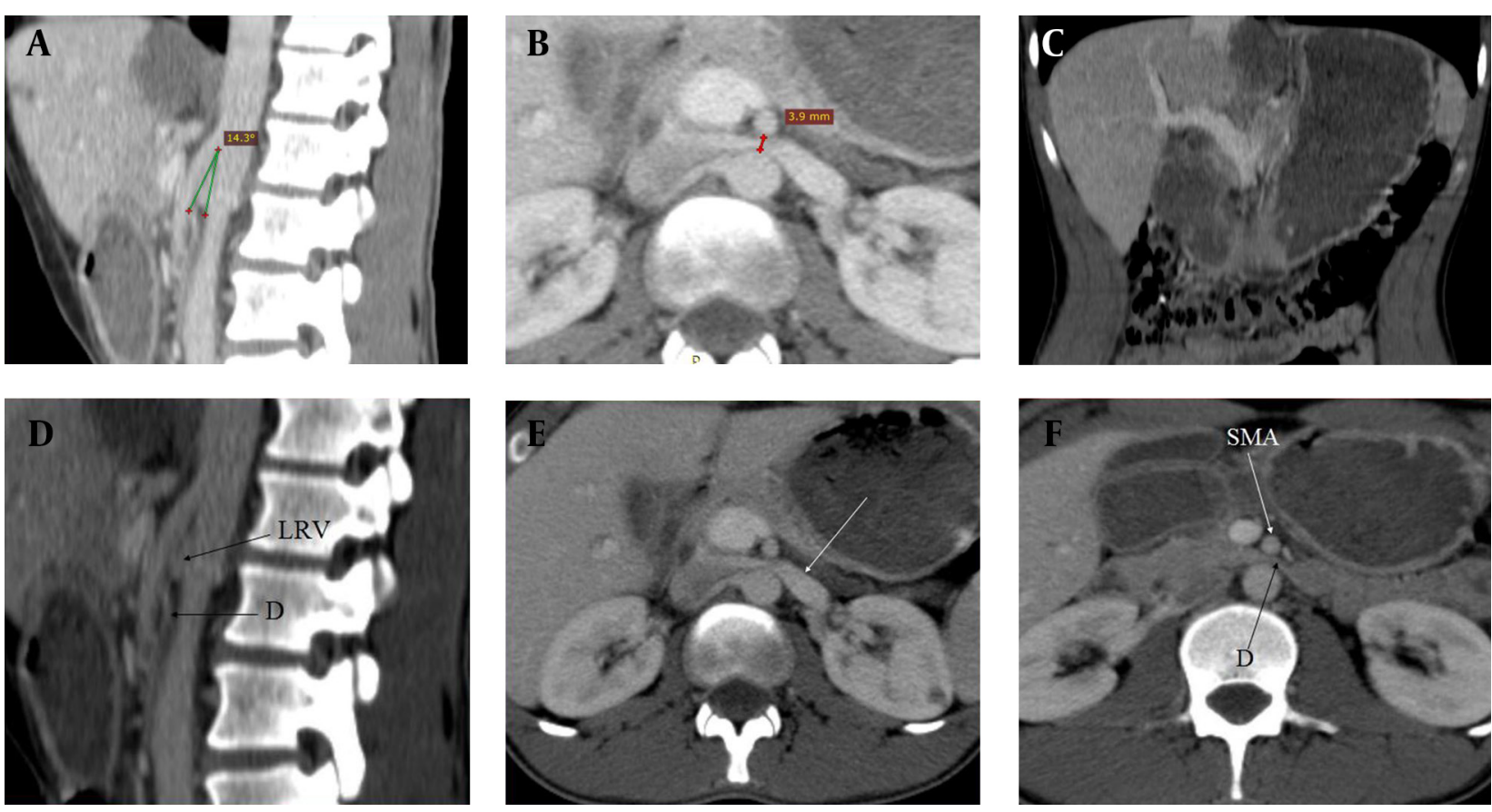

A) Sagittal CT scan showing the reduction of the angle (14 30) between the abdominal aorta and superior mesenteric artery. B) Axial CT scan showing decreased aortomesenteric distance (3,9 mm). C) Coronal CT scan showing dilatation of the stomach and proximal duodenum. D) Sagittal CT scan of abdomen showing duodenal compression between the abdominal aorta and superior mesenteric artery (D: Duodenum, LRV: Left renal vein). E) Axial CT scan of abdomen demonstrating duodenal compression between the abdominal aorta and superior mesenteric artery (D, Duodenum; SMA, Superior mesenteric artery). F) Axial CT scan showing dilated left renal vein owing to superior mesenteric artery compressing (white arrow).

(Figure 1B). Gastric and proximal duodenal dilatation was observed (Figure $1 C$ ) with the compression of the duodenal lumen between the AA and SMA (Figures $1 \mathrm{D}$ and $1 \mathrm{E}$ ). The LRV was also compressed by the SMA close to its origin, resulting in proximal dilatation (Figure F). The diagnosis of SMA syndrome accompanying NCS was established by these findings. The patient was treated conservatively through enteral nutrition support with a jejunostomy feeding tube. The therapy was effective in weight gain and pain reduction.

\section{Discussion}

SMA syndrome is a rare condition where the third part of the duodenum is compressed between the SMA and AA, leading to duodenal outlet obstruction. The incidence of SMA syndrome has been found to be between $0.013 \%$ and $0.3 \%$ of the general population (1). SMA syndrome is more common in females, and usually occurs between 35 and 45 years of age. However, it may also occur in older children and adolescents (2).

Clinical diagnosis of SMA syndrome is rather challenging owing to nonspecific character of the signs and symptoms in the majority of the patients, requiring a radiological diagnosis or confirmation of diagnosis. Upper gastrointestinal barium x-ray may demonstrate gastric and duodenal dilatation, or blockade of barium flow from duodenum to jejunum. An endoscopic gastroduo- denoscopy or CT scan of the abdomen could be helpful for confirmatory studies. These modalities may reveal the extrinsic compression of the third portion of the duodenum and the narrow angle between the AA and SMA (2).

Radiographically, SMA syndrome is characterized by several findings such as gastric and proximal duodenal dilatation with compression of the duodenum and dilatation of the left renal vein. In normal patients, the distance between the AA and SMA (aortomesenteric distance) is 10-34 mm with aortomesenteric angle of $28^{\circ}-65^{\circ}$ $(3,4)$. Angiographic studies have indicated that patients with SMA syndrome have an abnormal aortomesenteric angle $\left(6^{\circ}-22^{\circ}\right)$, and a shortened aortomesenteric distance (2-8 $\mathrm{mm})$ compared to normal patients. In a study using multidetector computed tomography (MDCT), the average aortomesenteric angle in patients with SMA syndrome was $13.5^{\circ}$, with an aortomesenteric distance of $4.4 \mathrm{~mm}$. Evidently, any absolute numerical cut-off is arbitrary, but some reports have advocated that an aortomesenteric distance $<8 \mathrm{~mm}$ and an angle $<22^{\circ}$ are suggestive of the diagnosis providing the right clinical setting (5).

The LRV normally passes between the AA and SMA, where it may be pressed, resulting in a variable degree of LRV obstruction. This compression of LRV between AA and SMA is entitled nutcracker syndrome (NCS) (6). The NCS is the clinical equivalent of nutcracker phenomenon (NCP) defined by a set of symptoms with significant alter- 
ations (7). While the terms NCP and NCS are sometimes used interchangeably in the literature, Shin and Lee accentuated that the nutcracker anatomy is not always accompanied with clinical symptoms and that some of the anatomic findings suggestive of nutcracker may stand for a normal variant or be accounted for by other conditions (8). Imaging, such as ultrasonography(US), Doppler US, CT, or magnetic resonance imaging (MRI), is required to diagnose NCS. Acute narrowing of the LRV at the level of SMA (beak sign), lateral (hilar) dilatation and the presence of collateral pathways at CT imaging can be helpful for the diagnosis of NCS. However, the diagnostic procedure (considering the reference standard for establishing the diagnosis of NCS) is invasive selective left renal phlebography with the measurement of the pressure gradient between the LRV and IVC $(2,6)$.

Our patient had decreased aortomesenteric distance and aortomesenteric angle, dilated stomach and proximal duodenum and dilated LRV. English literature search revealed one case in which SMA syndrome was found in concomitance with NCP (4). SMA syndrome accompanied by NCS, a rare collaboration, is the strong point of our study. Lack of measurement of the clinical variables is the weak point of our study. SMA syndrome should be considered in the differential diagnosis of duodenal obstruction such as duodenal hypoplasia, duodenal stenosis, duodenal atresia, peptic ulcer disease, congenital bands, intestinal malrotation, annular pancreas, and pre-duodenal portal vein (2). Most cases occur in patients with weight loss and reduction of fat stores around superior mesenteric artery because of a variety of reasons (surgery, malabsorption, trauma, etc.) (3). In the differential diagnosis of NCS, pathologies that cause pain, hematuria and proteinuria with an unexplained cause can be considered (6).

Conservative management based on the correction of electrolyte imbalance, and appropriate enteral or parenteral nutrition could result in symptomatic improvement and weight gain in most patients. Surgery, which may be considered if conservative treatment fails, involves the release of the Treitz ligament. During surgery, the ligament may be simply released or reconstructive approaches such as the open or close laparoscopic duodenojejunostomy, or Roux-en-Y procedure are chosen (2). In NCS management, supporting treatment is sufficient if the symptoms are minimal. Surgical or radiological treatments are indicated for severe pain, evident hematuria and renal functional deterioration (6).

SMA and NCS are rare vascular compression conditions. SMA should be considered in the differential diagnosis of high gastrointestinal tract obstruction. NCS should be considered in the differential diagnosis of patients with unknown cause of hematuria and flank pain. In conjunction with an appropriate clinical history, CT findings indicating decreased aortomesenteric angle and a shortened aortomesenteric distance can suggest the diagnosis for both conditions.

\section{Authors' Contributions}

Mikail Inal and Birsen Unal Daphan diagnosed the patient and prepared the manuscript. Mirace Yasemin Karadeniz Bilgili helped in the diagnosis.

\section{References}

1. Chan DK, Mak KS, Cheah YL. Successful nutritional therapy for superior mesenteric artery syndrome. Singapore Med $\mathrm{J}$. 2012;53(11):e233-6.

2. Barsoum MK, Shepherd RF, Welch TJ. Patient with both Wilkie syndrome and nutcracker syndrome. Vasc Med. 2008;13(3):247-50.

3. Felton BM, White JM, Racine MA. An uncommon case of abdominal pain: superior mesenteric artery syndrome. West J Emerg Med. 2012;13(6):501-2.

4. Vulliamy P, Hariharan V, Gutmann J, Mukherjee D. Superior mesenteric artery syndrome and the 'nutcracker phenomenon'. BM] Case Rep. 2013;2013.

5. Raman SP, Neyman EG, Horton KM, Eckhauser FE, Fishman EK. Superior mesenteric artery syndrome: spectrum of CT findings with multiplanar reconstructions and 3-D imaging. Abdom Imaging. 2012;37(6):1079-88.

6. Waseem M, Upadhyay R, Prosper G. The nutcracker syndrome: an underrecognized cause of hematuria. Eur J Pediatr. 2012;171(8):1269-71.

7. Kurklinsky AK, Rooke TW. Nutcracker phenomenon and nutcracker syndrome. Mayo Clin Proc. 2010;85(6):552-9.

8. Shin JI, Lee JS. Nutcracker phenomenon or nutcracker syndrome? Nephrol Dial Transplant. 2005;20(9):2015. 